Revue de droit comparé du travail et de la sécurité sociale

2 | 2021

La créativité du juge à l'épreuve des nouveaux problèmes de santé au travail

\title{
Croyances religieuses et soins transfrontaliers
}

CJUE 29 octobre 2020, aff. C-243/19, A c./ Veselibas ministriaja

Hélène Payancé

(2) OpenEdition

Journals

Édition électronique

URL : https://journals.openedition.org/rdctss/581

DOI : $10.4000 /$ rdctss.581

ISSN : 2262-9815

Éditeur

Centre de droit comparé du travail et de la sécurité sociale

Édition imprimée

Date de publication : 1 juin 2021

Pagination : 122-127

ISSN : 2117-4350

Référence électronique

Hélène Payancé, "Croyances religieuses et soins transfrontaliers ", Revue de droit comparé du travail et de la sécurité sociale [En ligne], 2 | 2021, mis en ligne le 01 juin 2021, consulté le 02 juin 2022. URL

http://journals.openedition.org/rdctss/581 ; DOI : https://doi.org/10.4000/rdctss.581

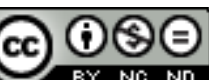

La Revue de droit comparé du travail et de la sécurité sociale est mise à disposition selon les termes de la Licence Creative Commons Attribution - Pas d'Utilisation Commerciale - Pas de Modification 4.0 International. 


\section{Croyances RELIGIEUSES ET SOINS TRANSFRONTALIERS CJUE 29 OCTOBRE 2020, AFF. C-243/19, A c./ Veselibas MiNISTRIAJA}

Dans cette affaire, le requérant est un ressortissant letton et témoin de Jéhovah. Son fils mineur souffre d'une malformation cardiaque et doit subir une opération du cœur. Cette opération nécessitant en Lettonie une transfusion sanguine contraire à ses convictions religieuses, le requérant a demandé au service national de santé la délivrance d'un formulaire S2, qui autorise son fils à bénéficier de soins programmés en Pologne, où l'opération est possible sans transfusion. Les autorités compétentes lettones ont refusé de lui délivrer cette autorisation, aux motifs que l'opération pouvait être effectuée en Lettonie et que seule la situation médicale d'une personne est prise en considération pour délivrer le formulaire susmentionné. L'enfant a finalement subi une opération du cœur en Pologne. Dans ce cadre, la Cour suprême lettone se demande si les services de santé nationaux pouvaient, sur le fondement de critères exclusivement médicaux, refuser la délivrance du formulaire S2 permettant la prise en charge des soins dans un autre État membre, en application de l'article 20 du règlement (CE) n`883/2004 et de l'article 8 de la directive 2011/24/UE, ou s'ils étaient également tenus de prendre en compte les croyances religieuses de l'assuré, en application de l'article 21 de la Charte des droits fondamentaux de l'Union.

En premier lieu, la CJUE relève que l'article 20, paragraphe 2, du règlement n̊883/2004, lu à la lumière de l'article 21, paragraphe 1, de la Charte, ne s'oppose pas à ce que l'État membre de résidence de l'assuré refuse d'accorder à ce dernier l'autorisation prévue à I'article 20, paragraphe 1, de ce règlement lorsque, dans cet État membre, un traitement hospitalier, dont l'efficacité médicale ne soulève aucun doute, est disponible mais que les croyances religieuses de cet assuré réprouvent le mode de traitement utilisé.

La Cour a indiqué, en second lieu, que l'article 8, paragraphes 5 et 6 , sous d), de la directive $n^{\circ} 2011 / 24 /$ UE, lu à la lumière de l'article 21, paragraphe 1, de la Charte, s'oppose à ce que l'État membre d'affiliation d'un patient refuse d'accorder à ce dernier l'autorisation prévue à l'article 8 , paragraphe 1, de cette directive lorsque, dans cet État membre, un traitement hospitalier, dont l'efficacité médicale ne soulève aucun doute, est disponible mais que les croyances religieuses de ce patient réprouvent le mode de traitement utilisé. Il en irait différemment si ce refus était objectivement justifié par un but légitime tenant au maintien d'une capacité de santé ou d'une compétence médicale, et constituait un moyen approprié et nécessaire permettant d'atteindre ce but, ce qu'il appartient à la juridiction de renvoi de vérifier.

Ainsi, le régime d'autorisation préalable peut être analysé dans le cadre du règlement n883/2004 (I), puis dans le cadre de la directive 2011/24/UE (II). 


\section{I - LE RÉGIME D'AUTORISATION PRÉALABLE DANS LE CADRE DU RÈGLEMENT N883/2004}

En application du règlement $n^{\circ} 883 / 2004$, le régime d'autorisation préalable est cantonné à des critères médicaux (A). Même interprété à la lumière de la Charte des droits fondamentaux, le règlement autorise l'État de résidence à ne pas prendre en compte les convictions religieuses de l'assuré en raison de la nécessité de protéger l'équilibre financier du système de santé national (B).

\section{A - UN RÉGIME D'AUTORISATION LIMITÉ À DES CRITÈRES MÉDICAUX}

Les règlements de coordination distinguent les soins inopinés ${ }^{1}$ reçus à l'occasion d'un séjour dans un État membre, des soins programmés reçus lors d'un déplacement à des fins médicales. À la différence des premiers, la prise en charge des seconds est soumise à un régime d'autorisation préalable dès lors qu'il s'agit de soins hospitaliers non ambulatoires ${ }^{2}$.

Le paragraphe 1 de l'article 20 du règlement n883/2004 dispose « qu'une personne assurée se rendant dans un autre État d'affiliation aux fins de bénéficier d'un traitement médical demande une autorisation à l'institution compétente». Le paragraphe 2 , première phrase, du même article prévoit que l'assuré qui a été autorisé à se rendre dans un État en vue de recevoir des soins « bénéficie des prestations en nature servies, pour le compte de l'institution compétente, par l'institution du lieu de séjour, selon les dispositions de la législation qu'elle applique, comme si elle était assurée en vertu de cette législation ». En d'autres termes, si la charge financière des soins pèse sur l'institution d'affiliation, elle s'opère à hauteur de ce que prévoit la législation du lieu de séjour où les soins sont reçus ${ }^{3}$. L'originalité de ce régime d'autorisation réside dans la limitation des possibilités de refus par l'institution d'affiliation.

Le paragraphe précité, dans sa seconde phrase, énonce ainsi que l'autorisation est obligatoirement accordée lorsque deux conditions cumulatives sont réunies. La première condition exige que les soins en question figurent parmi les prestations prévues par la législation de l'État membre sur le territoire duquel réside l'intéressé. La seconde condition requiert que les soins que l'assuré social envisage de recevoir dans un État membre autre que celui dans lequel il réside ne puissent, compte tenu de son état actuel de santé et de l'évolution de sa maladie, lui être dispensés dans le délai normalement nécessaire pour obtenir le traitement adéquat dans l'Etat membre de résidence ${ }^{4}$. En l'espèce, la première condition ne pose pas de difficulté. La prestation en cause, l'opération du cœur, est prévue par la législation lettone.

Pour apprécier la seconde condition, point litigieux de l'affaire, la CJUE indique que le régime d'autorisation préalable prévu à l'article 20 du règlement n $883 / 2004$ " prend exclusivement en compte la situation médicale du patient, et non pas ses choix personnels en matière de soins médicaux » et considère qu'« en l'occurrence, il est constant que l'opération en cause au principal était nécessaire, afin d'éviter la dégradation irréversible des fonctions vitales ou de l'état de santé du fils du requérant au principal, compte tenu de l'examen de son état et de l'évolution prévisible de sa maladie. En outre, cette opération

1 Règlement (CE) n883/2004, article 19.

2 CJCE 13 mai 2003, aff. C-385/99, Muller Fauré.

3 CJCE 23 octobre 2003, aff. C-56/01, Inizan.

4 CJCE 16 mai 2006, aff. C-372/04, Watts; CJUE 5 octobre 2010, aff. C-173/09, Elchinov ; CJUE 9 octobre 2014, aff. C-268/13, Petru. 
pouvait être effectuée en Lettonie au moyen d'une transfusion sanguine, et il n'existait pas de motif médical justifiant de recourir à un autre mode de traitement. Le requérant au principal a refusé cette transfusion au seul motif que ses croyances religieuses s'y opposaient et a souhaité que l'opération en cause au principal ait lieu sans transfusion, ce qui n'était pas possible en Lettonie ». Ainsi, la décision des autorités lettones de refuser la délivrance de l'autorisation est compatible aux règlements de coordination.

Il peut être intéressant de relever que, jusqu'à présent, alors même qu'elle se fondait sur des critères médicaux identiques, la CJUE n'avait pas totalement écarté la prise en compte d'éléments extra-médicaux. Ainsi, la Cour considérait, sans pour autant se référer aux choix personnels de l'intéressé, que la situation médicale devait être appréciée au regard des contraintes qu'elle faisait peser sur l'exercice de son activité professionnelle ${ }^{5}$.

L'absence de toute référence à l'activité professionnelle dans le présent arrêt signifiet-elle que tout élément autre que strictement médical est exclu du régime d'autorisation, ou simplement qu'en l'espèce la situation médicale ne présentait aucune difficulté liée à l'exercice de l'activité professionnelle? Les prochains arrêts de la CJUE permettront de connaître la réponse à cette question.

Même interprété à la lumière de la Charte des droits fondamentaux, le règlement n883/2004 autorise l'État de résidence à ne pas prendre en compte les convictions religieuses de l'assuré en raison de la nécessité de protéger l'équilibre financier du système de santé national.

\section{B - UN REFUS D'AUTORISATION JUSTIFIÉ PAR LA NÉCESSITÉ DE PROTÉGER L'ÉOUILIBRE FINANCIER DU SYSTÈME DE SANTÉ NATIONAL}

Lorsque l'État membre de résidence de l'assuré refuse d'octroyer l'autorisation préalable prévue à l'article 20, paragraphe 1, du règlement $n^{\circ} 883 / 2004$, cet État met en œuvre le droit de l'Union, au sens de l'article 51, paragraphe 1, de la Charte, de sorte qu'il est tenu de respecter les droits fondamentaux garantis par celle-ci, notamment ceux consacrés à son article 21, paragraphe 1, à savoir le principe de non-discrimination fondée sur la religion'.

L'interdiction de toute discrimination fondée sur la religion ou les convictions revêt un caractère impératif en tant que principe général de droit de l'Union. II peut être rappelé que ce principe exige que des situations comparables ne soient pas traitées de manière différente, et que des situations différentes ne soient pas traitées de manière égale, à moins qu'un tel traitement ne soit objectivement justifié. Une différence de traitement est justifiée dès lors qu'elle est fondée sur un critère objectif et raisonnable, autrement dit lorsqu'elle est en rapport avec un but légalement admissible poursuivi par la réglementation en cause, et que cette différence est proportionnée au but poursuivi par le traitement concerné ${ }^{7}$.

En l'espèce, la réglementation nationale en cause au principal est formulée de façon neutre et ne donne pas lieu à une discrimination directe fondée sur la religion.

5 CJCE 23 octobre 2003, aff. C-56/01, Inizan ; CJUE 9 octobre 2014, aff. C-268/13, Petru. Selon sa formule classique la CJUE indiquait qu'« aux fins d'apprécier si un traitement présentant le même degré d'efficacité pour le patient peut être obtenu en temps utile dans l'État membre de résidence, l'institution compétente » devait se demander si la situation médicale de l'assuré rendait «impossible ou excessivement difficile l'exercice d'une activité professionnelle ».

6 CJUE 11 juin 2020, aff. C-634/18, Prokuratura Rejonowa w Slupsku.

7 CJUE 9 mars 2017, aff. C-406/15, Milkova. 
La juridiction de renvoi relève que, à la différence des personnes dont l'état - ou celui de leurs enfants - requiert une intervention médicale telle que celle en cause mais qui ne sont pas témoins de Jéhovah, les croyances religieuses du requérant affectent le choix de ce dernier en matière de soins. En effet, pour les témoins de Jéhovah, l'interdiction des transfusions sanguines faisant partie intégrante de leurs croyances religieuses, ils ne pourraient accepter de subir une intervention médicale impliquant de telles transfusions. Étant donné que l'État membre de résidence ne couvre pas le coût d'un autre traitement, autorisé par leurs croyances religieuses, les dépenses occasionnées par ce traitement devraient être prises en charge individuellement par des personnes telles que le requérant au principal.

Cette situation fait apparaître une différence indirecte de traitement fondée sur la religion entre, d'une part, les patients qui subissent une intervention médicale avec transfusion sanguine dont les coûts sont couverts par la sécurité sociale de l'État membre de résidence et, d'autre part, les patients qui, pour des raisons religieuses, décident de ne pas subir une telle intervention dans cet État membre et de recourir, au sein d'un autre État membre, à un traitement auquel ne s'opposent pas leurs croyances religieuses, dont les coûts ne sont pas couverts par le premier État membre. Il s'agissait alors de savoir si cette différence de traitement est fondée sur un critère objectif et raisonnable.

La CJUE estime que cette différence peut être justifiée par la protection de la stabilité financière du système d'assurance maladie letton dans la mesure où le régime d'autorisation prévu par les règlements de coordination implique un remboursement intégral à la charge de l'État d'affiliation. Dans l'hypothèse où les coûts des soins dispensés dans l'État de séjour sont plus élevés que ceux des soins offerts dans l'État de résidence, l'obligation d'un remboursement intégral peut engendrer des surcoûts pour cet État. Si les motifs religieux de l'assuré devaient être pris en compte pour la délivrance de l'autorisation, ce risque de surcoût serait certes imprévisible, les convictions religieuses étant par nature subjectives, mais potentiellement important.

La Cour en déduit qu'un régime d'autorisation préalable ne tenant pas compte des convictions religieuses de l'assuré, mais axé sur une évaluation médicale objective, peut réduire ce risque d'atteinte à l'équilibre financier d'un système de santé national. Elle considère que cette mesure "satisfait à l'exigence de proportionnalité ". Ainsi, l'article 20, paragraphe 2, du règlement $n^{\circ} 883 / 2004$, lu à la lumière de l'article 21 , paragraphe 1, de la Charte, doit être interprété en ce sens qu'il ne s'oppose pas à ce que l'État membre de résidence de l'assuré refuse d'accorder à ce dernier l'autorisation prévue à l'article 20, paragraphe 1, de ce règlement lorsque, dans cet État membre, un traitement hospitalier, dont l'efficacité médicale ne soulève aucun doute, est disponible, mais que les croyances religieuses de cet assuré réprouvent le mode de traitement utilisé. En d'autres termes, la justification de la différenciation ne permet pas de caractériser une discrimination. Même interprété à la lumière de l'article 20, paragraphe 1, de la Charte, l'article 20, paragraphe 2, du règlement autorisait l'État de résidence à ne pas prendre en compte les convictions religieuses du patient.

Cet arrêt peut interroger sur l'appréciation de l'exigence de proportionnalité de la différence de traitement au but poursuivi. Le risque de surcoût affectant l'équilibre financier de la sécurité sociale n'existe que dans l'hypothèse où le coût des soins dans l'État membre d'accueil est supérieur à celui des soins réalisés dans l'État de résidence. Dans l'hypothèse contraire, un afflux de demandes d'autorisation dû à la prise en compte des croyances religieuses ne constituerait pas un risque pour l'équilibre financier de la sécurité sociale. Nous pouvons alors nous demander si le refus systématique de prendre en considération les convictions religieuses de l'assuré, quel que soit le niveau des législations en cause 
quant au coût des soins en matière de santé, n'est pas une exigence disproportionnée au regard de l'objectif poursuivi de stabilité financière.

Parallèlement au régime d'autorisation préalable dans le cadre du règlement n883/2004, la CJUE a étudié ledit régime dans le cadre de la directive 2011/24/UE.

\section{II - LE RÉGIME D'AUTORISATION PRÉALABLE DANS LE CADRE DE LA DIRECTIVE 2011/24/UE}

La Cour rappelle que le régime d'autorisation, dans le cadre de cette directive, est soumis à la libre prestation des services (A). L'équilibre financier du système de santé national n'étant pas menacé (B), la directive n'autorise pas l'État de résidence à ne pas prendre en compte les convictions religieuses de l'assuré.

\section{A - UN RÉGIME D'AUTORISATION SOUMIS À LA LIBRE PRESTATION DES SERVICES}

L'article 8 de ladite directive prévoit, à l'instar du règlement de coordination, que l'État membre d'affiliation ne peut refuser d'accorder une autorisation préalable si « les soins de santé ne peuvent être dispensés sur son territoire dans un délai acceptable sur le plan médical, sur la base d'une évaluation médicale objective de l'état pathologique du patient, de ses antécédents, de l'évolution probable de sa maladie, du degré de sa douleur, et/ou de la nature de son handicap au moment du dépôt ou du renouvellement de la demande d'autorisation ". L'État membre d'affiliation peut refuser d'accorder une autorisation préalable lorsque « les soins de santé peuvent être dispensés dans un délai acceptable sur le plan médical, compte tenu de l'état de santé du moment de chaque patient concerné et de l'évolution probable de sa maladie ».

L'existence d'une certaine proximité entre le régime d'autorisation issu de la directive et celui issu du règlement, fondés tous deux sur des critères médicaux, peut être relevée. Pour autant, la CJUE ne conclut pas, au regard de la Directive, à la légalité du refus par l'administration lettone de prendre en considération les convictions religieuses du patient.

La Cour rappelle que la directive a codifié sa jurisprudence relative à la liberté de prestation de services garantie par l'article 56 TFUE dans le domaine des soins de santé, aux termes de laquelle un régime d'autorisation constitue a priori une entrave à la libre circulation des patients, à moins qu'il poursuive un objectif légitime et soit proportionné à cet objectif. L'article 7, paragraphe 1, de la directive prévoit ainsi que « le régime d'autorisation préalable, y compris les critères, l'application de ceux-ci et les décisions individuelles de refus d'autorisation préalable, se limite à ce qui est nécessaire et proportionné à l'objectif poursuivi et ne peut constituer un moyen de discrimination arbitraire ni une entrave injustifiée à la libre circulation des patients».

Les autorités lettonnes mettaient en avant des objectifs de maîtrise des coûts et de garantie à un accès suffisant et permanent à une gamme équilibrée de traitements de qualité. Concernant l'objectif tenant au maintien d'une capacité de soins de santé ou d'une compétence médicale, prudemment, la CJUE renvoie au juge national le contrôle de la nécessité et de la proportionnalité, tout en relevant qu'à l'instar de l'article 20, paragraphe 2 , du règlement, l'article 8 , paragraphes 5 et 6 , sous d), de la directive ne tient compte que de la situation médicale du patient, laissant ainsi apparaître une cohérence d'interprétation entre la directive et le règlement.

Concernant l'objectif de maîtrise des coûts, la Cour estime qu'aucune raison financière ne justifiait le refus d'autorisation. 


\section{B - UN REFUS D'AUTORISATION NON JUSTIFIÉ PAR LA NÉCESSITÉ DE PROTÉGER L'ÉOUILIBRE FINANCIER DU SYSTÈME DE SANTÉ NATIONAL}

La Cour relève l'existence d'une différence systémique entre le système de remboursement mis en place par le règlement $n^{\circ} 883 / 2004$ et celui prévu par la directive 2011/24/UE. En effet, dans le cadre de ladite directive, la prise en charge des soins par l'État d'affiliation se fait à la hauteur non pas du montant prévu par la législation de l'État de soins, mais des coûts qu'il aurait pris en charge si les soins avaient été réalisés sur son territoire dans la limite des frais réellement dispensés. Il en résulte que, dans le cadre de la directive, l'État d'affiliation ne peut pas être exposé, en cas de soins transfrontaliers, à une charge financière supérieure à celle qu'il aurait à assumer si les mêmes soins étaient réalisés sur son territoire. Par conséquent, la Cour estime en l'espèce à juste titre, qu'aucune raison financière ne justifiait le refus d'autorisation et l'absence de prise en compte des convictions religieuses du patient.

Il peut être observé que l'alternative dans l'application des dispositions, prévue dans le considérant 30 de la directive selon lequel «soit la présente directive s'applique, soit les règlements de l'Union sur la coordination des systèmes de sécurité sociale s'appliquent », est démentie par cet arrêt dans lequel la CJUE admet l'application des deux textes puisque leur interprétation par la juridiction nationale était demandée. Cette coexistence de textes peut, comme le cas présent, se révéler profitable à l'assuré. Alors que le refus d'autorisation est justifié par le règlement, il peut être remis en question sur le fondement de la directive. Toutefois, cette coexistence peut être critiquée en termes de cohérence du droit de l'Union européenne. 